\section{Briginal âtricles.}

THE CAUSES AND TREATMENT OF PARALYTIC DISLOCATIONS AND SUBLUXATIONS OF THE HIP-JOINT.

BY JAMES WARREN SEVER, M.D.,

Junior Assistant Surgeon, Children's IIospital, Boston; Member American Orthopedic Association.

THE following paper is the result of the careful study of a large number of cases of subluxations and dislocations of the hip-joint due to infantile paralysis. It is apparently a much more common condition than has been realized and leads to most severe deformity and disability unless recognized and treated. I am indebted to the staff of the Massachusetts State Hospital School for Cripples at Canton, the Children's Hospital and the Peabody Home for Crippled Children for the privilege of observing these cases, and to the X-Ray Department of the Children's Hospital for some of the x-rays accompanying this article. I am also indebted to Dr. Conrad Bell, of Waltham, for one case seen in consultation with him, and to Dr. Allison, of St. Louis, for one case seen with him at the Children's Hospital, St. Louis, Mo.

\section{LITERATURE.}

In a review of the literature of this subject, there were many points of interest.

Reclus. $^{1}$ - Reclus states that two conditions are necessary for the production of a dislocation, namely, (1) atrophy of a certain group of muscles; and (2) the integrity of their antagonists. He states that at the hip the iliac dislocations are the more frequent, due to an unopposed contraction of the adductors. He reports four cases, as follows:

CAse 1. Paralysis at six months of age, followed by an iliac dislocation of the hip.

CASE 2. Paralysis at seven years, where the patient at twenty-five showed an iliac dislocation due to persistence of the adductors with three inches shortening of the leg.

CAse 3. Paralysis at two and one-half years. Seen at eighteen, no dislocation on account of complete paralysis of all muscles.

CAse 4. Paralysis at sixteen months. Seen at three years, complete paralysis and no dislocation.

Reclus believes in trying to reduce the dislocation first, before doing tenotomies. He states that the more severe and complete the paralysis, the easier will be the reduction.

Karewski. ${ }^{2}$ - Karewski reports two cases, one infrapubic and one on the ramus of the pubes. These were due to the contraction of the tensor vaginæ femoris and quadriceps. The psoas, iliacus, external rotators and flexors of the thigh still retained their functions; with the paralysis of the abductors and extensors. The patients had never walked. The legs were in permanent flexion, external rotation and abduction. Following operation, in one case there was a poor result, for the head could not be replaced in the acetabulum, while in the other the great trochanter was found in the acetabulum. Following a correction of this condition and the reduction of the dislocation there was a good functional result.

Bonnet and Parire. - In studying the etiology of dislocation of the hip, Bonnet and Parire, quoted by Stimson, ${ }^{3}$ showed that by forcible injection of liquid into the cavity of the hip-joint the articular surfaces could be separated one-eighth to one-fourth inches. Fluid in the joint causes the leg to become flexed. This flexed position relaxes the $Y$ ligament, and if the capsule is filled with liquid, the head of the femur can leave the cotyloid cavity without creating a vacuum, for the liquid takes its place, and thus a great obstacle to dislocation is removed, namely, atmospheric preasure.

Nicoletn: ${ }^{4}$ - Nicoletti reports a case with infantile paralysis of the left leg. The left leg was in a position of abduction and external rotation, and there existed a subluxated hip. There was also a flexor contracture of the knee-joint, with equinus of the foot. After tenotomy of the fascia lata and rectus femoris and partial dissection of the gluteii from the great trochanter, an arthrodesis of the hip-joint was done. A new pan was chiseled in the upper anterior part of the old acetabulum which had nearly disappeared, and the head adapted to this pan. The result was a complete ankylosis, with a short leg, but symmetrical with the other.

Vulpius. ${ }^{5}$ - Vulpius believes that arthrodesis should be reserved for definite conditions, and only when certain that the advantages to be gained are greater than the damage already existing. He believes that forward dislocations are frequent in cases which have to go about on the hands in a sitting position.

Böcker. ${ }^{6}$ - Böcker in a paper entitled, "Are There Really Forward Dislocations of the Hip?" states that there are about twice as many infrapubic dislocations reported as iliac dislocations. $\mathrm{He}$ believes that these diagnoses of forward dislocations are not supported in the literature by $x$-ray findings, and gives a list to date of reported cases, to which he added three cases of iliac dislocations and one of infrapubic dislocation. Böcker does not believe in a permanent paralytic dislocation of the hip forward, but calls the condition a subluxation with near-arthrosis.

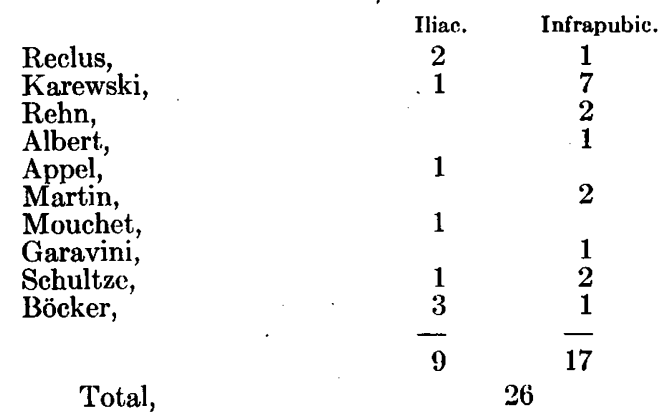

Cramer. ${ }^{7}$ - Cramer speaks of the influence of excess fluid in the joint as a cause of dislocation, and also dislocations following typhoid, measles, scarlet fever and diphtheria. He quotes Graff, 
who believes that in these conditions there may be an inflammatory softening of the upper part of the acetabulum. He also quotes Seeligmüler's theory as to the cause of paralytic dislocations, namely, that they are due to the influence of the contraction of unopposed muscles, combined with body weight in weight bearing, especially so in the production of the dislocations of the iliac type. He gives one case of his own in which the child was completely paralyzed at eighteen months. The paralysis involved the whole body and extremities. At twelve years of age the left leg was flexed and contracted, the quadriceps and adductors paralyzed, the tensor vaginæ femoris, sartorius and vastus externus weak, with a strong ilio-psoas muscle. Right leg, paralysis of gluteii, extensors atrophied, but tensor vaginæ femoris still had power and the adductors were strong. The child had never walked. X-rays showed the head of the femur on the right displaced upward and backward on the ilium. The upper edge of the acetabulum was flat, but the cavity not lengthened. There was slight lateral displacement of the epiphysis of the head of the femur. The x-ray of the left hip showed the head displaced backward and downward, with a flattening of the acetabulum. This left leg he attempted to replace after cutting the contracted muscles, but was not successful.

He quotes two cases reported by Rehn, one in which the adductors and quadriceps were paralyzed and where the head of the femur was in the region of the obturator foramen. This patient died of an intercurrent disease, and the autopsy showed an alteration in the bones of the pelvis causing asymmetry, the acetabulum was pushed upward, the bones were soft, and there was marked atrophy at the points of insertion of the paralyzed muscles, with hypertrophy at the points of insertion of the non-paralyzed muscles.

The second was a case of double iliac dislocation in a child of sixteen years which showed by x-ray examination the head of the femur displaced upward and backward articulating in a new acetabulum. A double arthrodesis was done in this case, and eight weeks after the operation the patient could walk with assistance.

Cramer reports one other case in a girl of fourteen years with a paralytic iliac dislocation on the left, with normal gluteii, ilio psoas and quadriceps muscles.

Reidinger. ${ }^{8}$ - Reidinger, in the best paper so far on the subject, speaks of iliac dislocations. He quotes some of the other writers, and states that from the type of deformity present one cannot tell which muscles are paralyzed, owing to the complicated mechanical causes. A sitting position pushes the head of the femur forward and so wears off the upper and anterior portion of the acetabulum. He speaks of the infrequency of infrapubic dislocations and states that with Schultz's cases of a fifteen-year-old boy and a nineteenyear-old girl and seven cases of Karewski's there are only seven other cases reported. He states that Rehn believes the condition due to a wandering acetabulum and that it is not a true disloca- tion. He quotes Rehn as believing in the method of bloodless reduction, while Karewski believes in an open operation after cutting the contracted tissues. He quotes Hoffa as stating that it is impossible to replace old cases of long-standing paralytic dislocations of the hip.

Schultz, quoted by Reidinger, made two unsuccessful attempts of reduction. In one case, with complete paralysis of the left leg after tenotomy of the tensor vaginæ femoris, on attempting reduction the neck of the femur broke and the head of the bone stayed in the acetabulum. He later did a resection of the knee on the same leg. He obtained a functional leg with $20 \mathrm{~cm}$. shortening, so that the patient could walk with a cane, whereas before operation he could not walk at all.

In the second case there was a complete paralysis of the right leg, with an infrapubic dislocation, a contracted knee and an acquired club foot. On the left there was an iliac dislocation, with a club foot. He corrected the contractions by tenotomies and did a resection of each kneejoint. No attempt was apparently made to correct the condition of the hip. The second case could not walk without crutches, but both were considerably improved. He believes that resection of the knee-joint is indicated in cases of total paralysis of leg muscles.

Lovett and Lucas. $^{9}$ - Lovett and Lucas, in a study of six hundred and thirty-five cases of infantile paralysis observed at the Children's Hospital in Boston in 1908, report that the anterior muscles of the thigh are affected much more frequently than the posterior ham-string muscles, and that the sartorius frequently escapes when the quadriceps femoris is paralyzed. At the time of the report but two cases were found in which the hip was dislocated.

SUMMARY OF CONCLUSIONS FROM THE LITERATURE.

(1) Presence of unopposed contractures, with and without weight bearing, of great importance.

(2) Predominance of dorsal type of dislocation.

(3) Infrequency of infrapubic or forward dislocation.

(4) Favorable prognosis for a useful leg provided the case is of not too long standing, so that the contractions can be stretched or divided and the dislocation reduced.

(5) An arthrodesis of the hip-joint has been found of use.

\section{MECHANISM.}

These subluxations and dislocations of the hipjoint are the direct result of the lack of proper muscular support due to infantile paralysis and are associated with certain secondary bony and cartilaginous changes in the acetabulum and head of the femur, due to atrophy and gravity, and of which I shall speak more fully later. The condition may be either incomplete or complete, depending on the severity and extent of the paralysis and the duration of the disease. The condition usually occurs only in cases of long-standing paralysis, that is, of over a year. Weight bearing may 


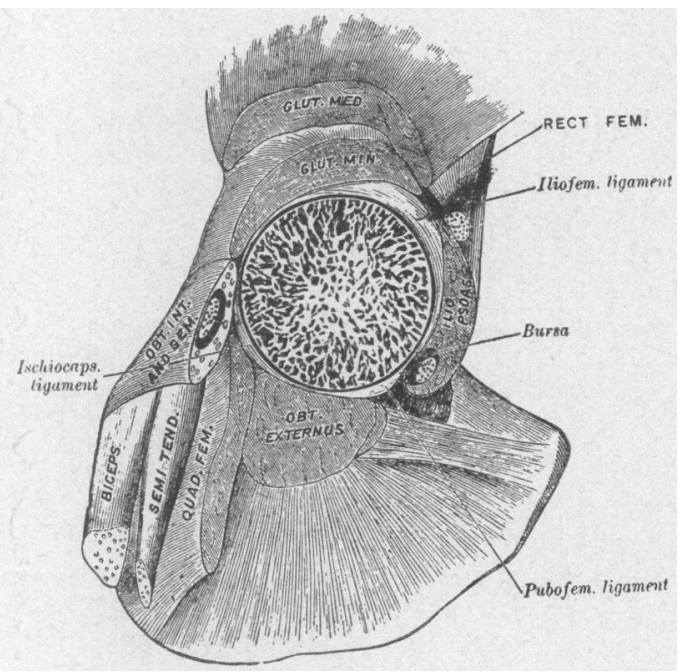

Fia. 1.

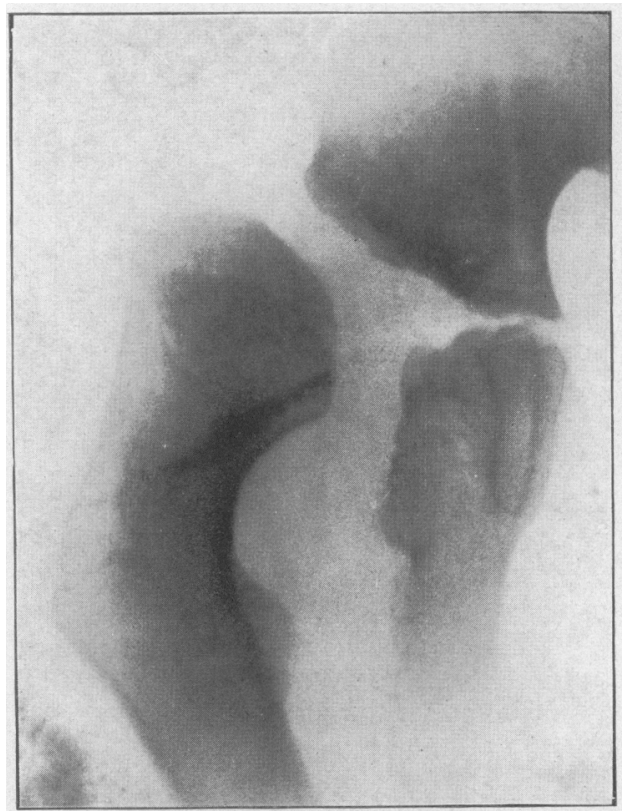

Fic. 3.

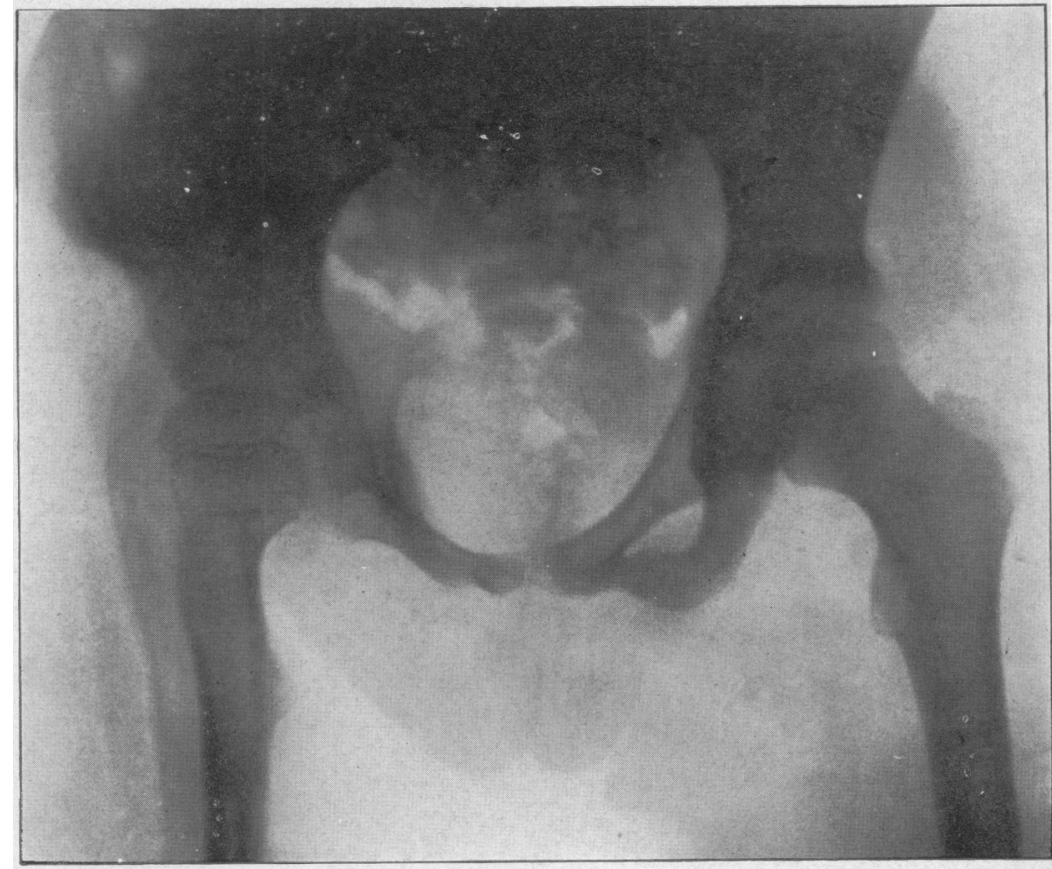

Fli. 2.

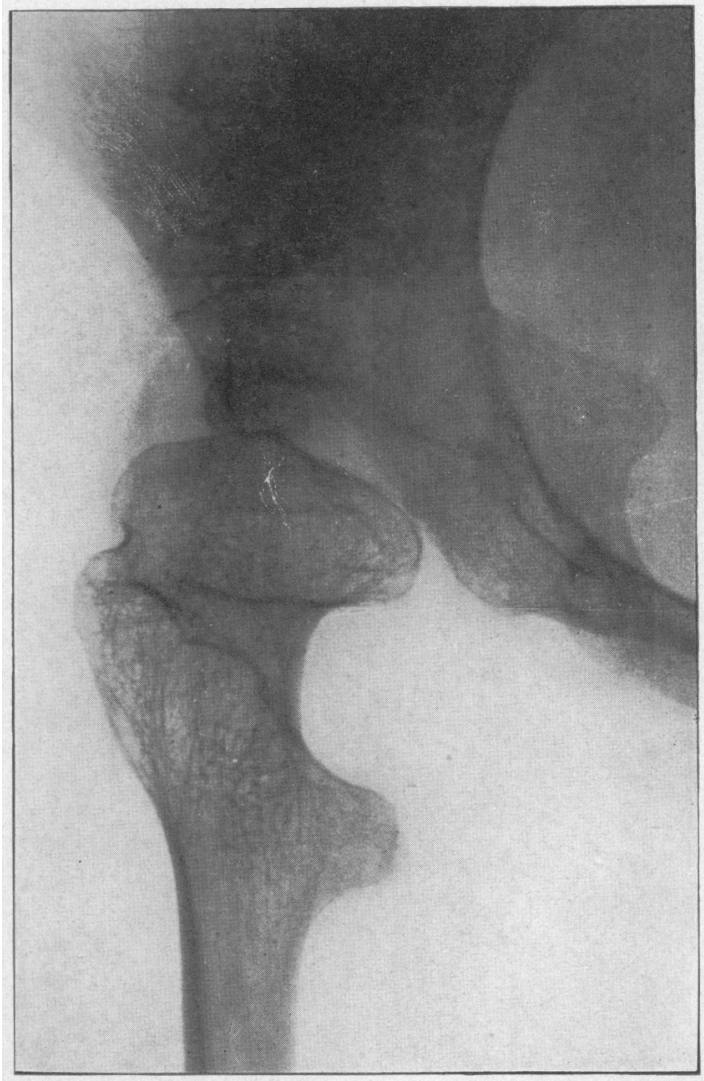

Firi. 4.

The Boston Medical and Surgical Journal as published by 


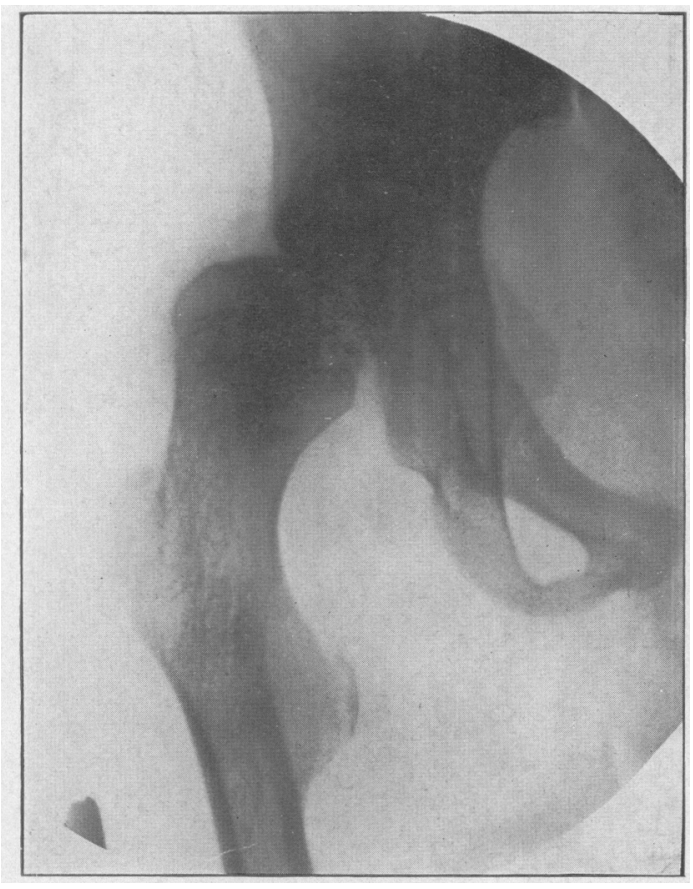

Fig. :5.

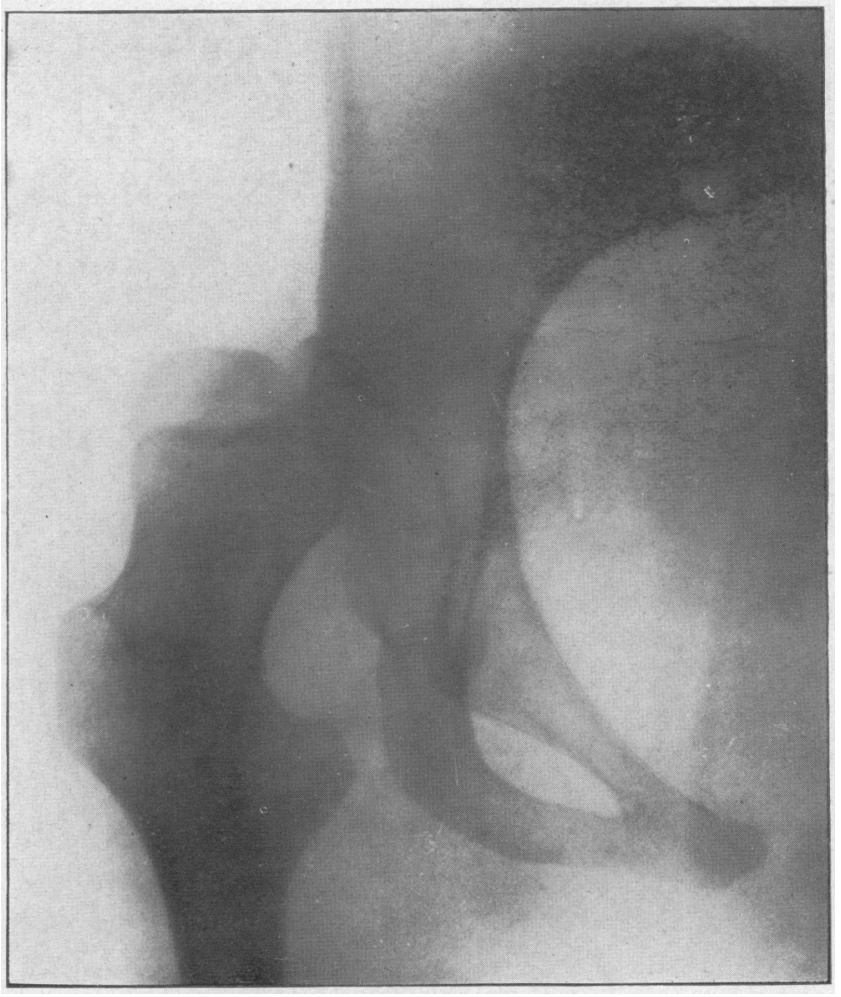

Fig. 6.

The Boston Medical and Surgical Journal as published by 
be an important factor in its production, but is not essential.

To differentiate clearly between the terms "subluxation" and "dislocation" I will state that a subluxation of the hip, as used in this paper, means a hip-joint so relaxed that it is possible by manipulation to dislocate partially or completely the head of the femur from the acetabulum, but does not mean that the head of the femur lies habitually outside of its socket.

Dislocation, on the other hand, means, as used here, a permanently displaced head, which it may or may not be possible to replace in the acetabulum.

Unopposed contractions of the non-paralyzed muscles are important factors in the production of this condition, such as the unopposed contraction of the adductors and the tensor vaginæ femoris muscles. When paralysis involves all the muscles about the hip-joint the joint becomes loose and the head of the femur may be displaced and replaced at will, but when only a few of the muscles are paralyzed, the unopposed contractions of the others may lead to permanent displacement of the head of the femur. For example, if the posterior muscles of the hip are paralyzed and the adductors remain active, the dislocation may be dorsal, while if the adductors are paralyzed and the gluteii remain, the dislocation may be forward on to the pubes. In the dorsal form the dislocation gradually takes place owing to the support of the peri-trochanteric muscles being withdrawn from the capsule, which gradually stretches and yields, owing to the pressure of the head on the upper and posterior portion of the joint, until a dislocation occurs. In the case of an anterior dislocation, the adductors and psoas are paralyzed so that the gluteii and peri-trochanteric muscles gradually push the head forward.

The most common form of subluxation seen is that in which the head of the femur is dislocated upward and backward on to the dorsum of the ilium, where it can be both seen and felt, and is often mistaken for a high trochanter. Shortening of the leg associated with a limp is present. Cases of pubic and infrapubic dislocation have been reported, but I have seen but one, that is, a case of infrapubic. In practically all of the cases the head of the femur can be slipped in and out of the acetabulum to a greater or less extent.

Before going further into the causation of these dislocations it is essential to understand the anatomy of the hip-joint with its surrounding muscles and their actions.

\section{ANATOMY.}

The hip-joint is a ball and socket joint, and under ordinary conditions of muscular contraction a dislocation is an impossibility. The acetabulum or socket is deepened by the cotyloid ligament, a firm elastic cartilage which covers its bony rim, forming an elastic instead of a rigid cushion, to check too free motion of the femur, and which, as it fits on tight to the globular head, constitutes a sucker, enabling atmospheric pressure to maintain the integrity of the joint. The bony surfaces are not, however, held together by atmospheric pressure alone, for the insertion of the gluteus minimus, iliacus and psoas magnus muscles are such as to enable them to aid in making tense the capsule, and giving security to the joint. The capsule, whose attachment is of a stronger nature than the connection between the epiphysis and diaphysis, arises from the entire circumference of the acetabular rim where it is thickest, and is attached to the anterior intertrochanteric line in front of the neck of the femur and above the posterior inter-trochanteric line behind. Although strong, it is so loose that it allows all ordinary motions of the joint without becoming tense, and hence plays no part in holding the joint surfaces together.

There is also the ligamentum teres, which runs from the head of the femur to the base of the cotyloid notch. In some cases this ligament is rudimentary, and in others is absent. The ligament is made tense when the leg is semi-flexed, adducted and rotated outward. It is, however, of very little use as a ligament.

\section{NERVE SUPPLY.}

The nerves which supply the muscles of the hip and thigh are as follows:

The anterior crural, from the third and fourth lumbar nerves.

The obturator, from the second, third and fourth lumbar nerves.

The sacral plexus, which is formed by the lumbosacral cord, the anterior division of the three upper sacral nerves and part of the fourth.

The sciatic nerve, from the upper cord of the sacral plexus.

The superior gluteal nerve, from fourth and fifth lumbar nerves and first sacral nerve.

The inferior gluteal nerve, from lumbo-sacral cord and first and second sacral nerves.

\section{MUSCLES.}

The separate muscles are supplied by the following nerves:

Psoas, by the anterior branches of the lumbar nerves.

Iliacus, by the anterior crural.

Tensor vaginæ femoris, by the superior gluteal nerve.

Rectus, by the anterior crural.

Adductors, by the obturator.

Pectineus, by the obturator and anterior crural.

Adductor magnus, by the obturator and great sciatic.

Gluteus maximus, by inferior gluteal from sacral plexus.

Gluteus medius, by superior gluteal.

Gluteus minimus, by superior gluteal.

Pyriformis, gemelli, obturator internus and quadratus femoris, by branches from the sacral plexus.

Obturator externus, by obturator nerve.

Hip-joint, by articular branches of obturator nerve.

See Fig. No. 1. 
MUSCLES OF THE THIGH AND HIP AND THEIR RELATIONS TO THE HIP-JOINT.

The muscles in relation to the hip-joint are, in front, the psoas and iliacus; above, the reflected head of the rectus and gluteus minimus, the latter being closely adherent to the capsule; internally, the obturator externus and pectineus; behind, the pyriformis, gemellus superior, obturator internus, gemellus inferior, obturator externus and quadratus femoris.

The movements of the hip-joint are very extensive, and the various motions are secured by the action of the following muscles:

The muscles which flex the femur on the pelvis are the psoas, rectus, iliacus, sartorius, pectineus, adductor longus and brevis, and the anterior fibers of the gluteus medius and minimus. Extension is obtained largely by the gluteus maximus, assisted by the ham-string muscles, adduction by means of the adductor longus, magnus and brevis, the pectineus and gracilis, and abduction by the gluteus maximus, medius and minimus. Inward rotation is obtained by the action of the anterior fibers of the gluteus medius, the gluteus minimus and tensor vaginæ femoris, and outward rotation by the posterior fibers of the gluteus medius, the pyriformis, obturator externus and internus, gemelli, quadratus femoris, psoas, iliacus, gluteus maximus, the three adductors, pectineus and the sartorius. It is essential to understand the action of these separate muscles thoroughly, so that in any given case one may determine by the voluntary motions of the leg at the hip which muscles are paralyzed and which are not, and, therefore, so determine, at least partially, the causes of the types of dislocation or subluxation present. The treatment necessarily depends on this accurate analysis.

\section{EXPERIMENTS ON CATS AND RABBITS.}

I am indebted to Dr. Nichols, of the Laboratory of Surgical Pathology, Harvard Medical School, for the opportunity to carry out these experiments.

It became a matter of considerable interest to me to determine if possible the mode of procedure in the establishment of these subluxations, and I, therefore, decided to try to produce them artificially in animals. First, I attempted to dislocate a hip in rabbits and cats, but, owing to the firm support of the ligaments and muscles, besides the unusually deep acetabulum, the short neck of the femur, which is at very nearly a right angle to the long axis of the shaft, and the high great trochanter, I found it impossible, even when using great force.

I then decided to divide certain nerves singly and together in separate animals so as to produce a condition of paralysis of the hip and leg muscles, somewhat analogous to that seen in anterior poliomyelitis. This condition I could not reproduce exactly, for in cutting the nerve trunks I not only destroyed the motor impulses, but also the sensory. However, I did not consider this latter condition a very great drawback. These following experiments are interesting, but not conclusive.
The anatomical relations and the applications of muscular force and weight bearing are very different in cats and rabbits as compared to a human hip-joint, and these experiments were undertaken more to see what might be produced than with the hope of really obtaining a dislocation. In November of 1910, I therefore operated on four rabbits and five cats.

In rabbit No. 1 , one inch of the great sciatic nerve was resected above the great trochanter. This animal died too soon after the operation for it to have had any effect.

Experiment No. 2. Rabbit. Sciatic resected; died within a few days.

Experiment No. 3. Rabbit. Sciatic resected Nov. 3, 1911. Killed Feb. 24, 1911. X-rayed. Showed slight bony atrophy on right. No dislocation. No changes in head or neck of femur, no trophic disturbance.

Experiment No. 4. Rabbit. Sciatic divided Nov. 3, 1910. Killed Feb. 23, 1911. Shows large trophic ulcer on right leg over os calcis. Leg much atrophied. Can apparently dislocate and replace hip. Some crepitus about hip-joint. X-ray shows a fracture of the acetabulum with bony atrophy of shaft of femur. Knee-joint shows beginning disintegration similar to the condition seen in locomotor ataxia. Head and neck of femur apparently like that of normal leg.

The five cats all lived about three months, long enough to show changes in the joint were any going to occur. On careful dissection and inspection no changes were visible, nor could the hip be dislocated. All of the cats but one had the anterior crural divided, in the other the great sciatic was divided.

\section{DESCRIPTION OF CASES.}

Author's Cases.

$\begin{array}{lr}\text { Boys, } & 8 \\ \text { Girls, } & 18 \\ \text { Total, } & \frac{26}{}\end{array}$

Relaxed but not subluxated,

Iliac subluxations,

Infrapubic (obturator),

3 early cases

In reporting these following cases it is manifestly impossible to reproduce all of the $x$-rays, but certain ones will be shown, and others which are characteristic and demonstrate the principal bony changes will be described. The cases are described below in detail in the order of the degree and importance of the deformity.

Case 1. D. S. Girl. Came to hospital in November, 1907, when nine months old, with a history of having had a paralysis of the right leg for one month. At that time the abductors and adductors of the thigh were very weak. The paralysis of the extensors and flexors of the thigh was not complete. She could extend the lower leg on the thigh and partially extend the thigh on the body. On Nov. 15, 1909, the condition was as follows: There was slight capsular relaxation of the hip-joint, with a shortening of an inch in length of the whole leg, due to atrophy of the leg. She walked with a bad limp, and showed a calcaneus of the right foot. This calcaneus was corrected in the fall of 1910 , and an x-ray taken in September, 1910, showed the 
following condition of the hip: Marked coxa valga on right, considerable atrophy of great trochanter, marked atrophy of whole neck and shaft of femur and whole right side of pelvis, acetabulum smaller on right than on left, no dislocation of hip-joint.

This case and Cases 2, 3 and 4 show the beginning stage of the various conditions which eventually lead to dislocation. The coxa valga is a constant condition in these cases and is apparently a pure gravity deformity in a dangle leg. In the early stages the shortening of the leg from atrophy of disease and disuse causes the child to abduct when walking, so that there is no dislocation of the hip in weight bearing, but as the atrophy goes on, accompanied in many cases by the unopposed contractures of the healthy muscles, a dislocation occurs, which in weight bearing is accentuated. This leads directly to a very short leg on the affected side, with considerable discomfort and disability. With the head of the femur out of the acetabulum so much of the time, especially in weight bearing, there occur secondary changes in the acetabulum resulting in a flattening, and consequently a shallower socket, an elongation of the acetabulum in an upward and outward direction, or a so-called "traveling acetabulum," with marked atrophy or absorption of the upper edge or shelf of the hip-joint. The joint capsule and ligamentum teres also stretch.

Case 2. J. A. Boy. Came to hospital Dec. 4, 1907, age three years. Duration of paralysis at that time was twelve weeks. There was complete paralysis of the left leg. In September, 1910, he was seen again, when the condition of the left hip as shown by x-ray was as follows: Marked coxa valga, very shallow sloping acetabulum, with partial subluxation of head of femur, considerable atrophy of femur and left side of pelvis. Walked only with a caliper splint. Limped badly, although in weight bearing the hip did not dislocate. This case at present is convalescing from some tendon operation on his lower leg muscles, a few of which were not affected. His hip so far does not need an operation. The hip cannot be dislocated manually.

CASE 3. S. W. Boy. Age three and one half at entrance to clinic in September, 1907. Duration of paralysis, two years. Shortening of right leg with almost total paralysis of right thigh muscles. There was no dislocation of the hip at that time. The gluteal group were mostly affected, the quadriceps were very weak or absent, the ham strings and adductors were of good strength. There was two inches shortening. On Oct. 26, 1910, age six and one half, there was one and one-half inches shortening. The trochanter was at Nélaton's line, and the boy could move the thigh freely, but there was some loss of muscle power. The $\mathrm{x}$-ray on that date showed a marked coxa valga, with a small shallow atrophied acetabulum, an atrophied right pelvis and a very small femur. The hip could not be dislocated, but the motion was much more free in all directions than was normal.

Case 4. M. H. Girl. (See Figure No. II.) Age cleven. Patient of Dr. R. W. Lovett. Paralysis of left leg since several months of age. Condition in December, 1910, as follows: Walked with a bad limp and drop on left. Hip did not slip out in walking owing to degree of abduction necessary to get leg to ground. Shortening one and one-half inches. Gluteii and extensors of thigh paralyzed, slight power in adductors, abductors and rotators of thigh. Tensor vaginæ femoris moderately contracted so that leg is pcrmanently flexed about twenty degrees. The hip could be slipped in and out of the acetabulum and moved upward and backward. The $\mathrm{x}$-ray showed the usual atrophy of pelvis and femur, with a marked coxa valga and a shallow, elongated and flattened acetabulum. This child can walk fairly well with a lift on the shoe and a supporting splint which bears on the pelvis to prevent the side drop.

CASE 5. E. D. Boy. Age nine. Paralyzed at two years of age. Shows a paralysis of left leg, with no power in any muscles except the adductors and tensor vagina femoris, which are contracted. Has had an arthrodesis of the left knee-joint to assist walking. Considerable shortening of leg. X-ray taken on March 17, 1910, shows marked coxa valga of neck of left femur, atrophy of greater and lesser trochanters. Head of femur not dislocated or subluxated, but distracted. Capsule loose, and by manipulating the femur the head can be slipped in and out of the acetabulum. This subluxation occurs usually by flexing and adducting the thigh on the abdomen. He walks fairly well, with a moderate limp. Case 6. F. C. Boy. Age ten. Paralysis of both legs for six years since four years of age. At present boy cannot walk without crutches or use legs at all. Has bad flexion deformity of both legs, with marked contraction of tensor vagina femoris and adductors of both legs. With child on the back the right hip is subluxated, with the head carried upward and backward. The left is apparently in the acetabulum. By manipulation of the right thigh the head can be replaced and dislocated at will. X-ray taken March 17, 1911, shows the right hip dislocated upward and backward on the ilium. The left hip is not dislocated. There is the usual coxa valga with atrophy of the shaft and trochanters. The acetabulum is very shallow, and is apparently filled up with cartilage.

CASE 7. N. H. Girl. Age fifteen. Paralysis since six years of age. Pelvis shows marked tilt forward, with hips permanently flexed to fifty degrees. Flexion and subluxation of both knees from contraction of ham strings. Marked contraction of tensor vaginæ femoris and adductors of both legs. Can walk only by aid of caliper splints and crutches. The right hip can be partially subluxated on to ilium. The leg cannot be raised off of the bed, and there is practically no muscle power in the leg. The gluteii are paralyzed. The left leg shows no muscle power. Paralysis of adductors, contraction of tensor vaginæ femoris marked. The hip can be partially subluxated on to ilium. X-ray taken March 17, 1911, with patient on the back, shows right hip in and the left out and behind the acetabulum. There is no coxa valga. There is a marked flexion of the pelvis with distortion. No great atrophy of the trochanter, but marked atrophy of the shaft of the bone.

CAsE 8. Girl. Age about eleven. Paralysis of right leg for about two years. Seen with Dr. Allison at the Children's Hospital, St. Louis. There is a good deal of atrophy of the leg, the paralysis affecting the extensors, rotators and gluteii of the thigh, and the anterior muscles of the lower leg. The adductors and tensor vaginæ femoris are moderaetly contracted. In walking the child has to abduct the leg on account of about one and one-half inches shortening so as to get the leg to the ground, and so engages the head of the femur in the acetabulum. When the child lies down the hip can be easily dislocated and replaced, but the process is slightly different from the usual one. In the greater number of the cases, by adducting, flexing and inwardly rotating the thigh, the hip can be dislocated and slipped upward and backward on to the ilium. In this case the leg had to be abducted, flexed and rotated 
outward to produce dislocation, when the head apparently slipped upward and behind the acetabulum.

The x-rays of this case showed practically no coxa valga. There was a well-shaped globular head to the femur, but a shallow atrophied acetabulum. The $x$-ray taken with the femur dislocated showed the head directly behind the acetabulum.

Case 9. N. M. G. Boy. Age fourteen. Paralysis since July, 1908. Both legs paralyzed, followed by contractions of the flexors, which have been corrected by means of plaster of Paris casts during the past year, so that he can now be fitted with calipers and crutches. Seen March, 1911. Both hips much relaxed, can be casily subluxated upward and backward, adductors and tensor vaginæ femoris much contracted and unopposed, lower legs flexed permanently on thighs and thighs flexed and adducted on abdomen.

CASE 10. L. B. Boy. Age seven. Paralysis since eight months of age. Lies with left leg outwardly rotated and abducted, moderately flexed at the hip. Tensor vaginæ femoris contracted. By manipulations it is possible to slip the hip out and into the joint, the head being displaced upward and backward. Considerable relaxation of joint capsule.

CASE 11. W. P. P. Boy. Age eight and one half. Infantile paralysis, right leg, for past year and a half. Complete paralysis of thigh muscles. Right hip is partially subluxated. Is wearing splints and using crutches.

The clinical findings in the cases which I was able to observe at the Massachusetts State Hospital School at Canton I was unable to confirm by means of $x$-rays.

CASE 12. Girl. Age seventeen. Infantile paralysis for past year and a half, complete of right leg and considerable paralysis of left hand and shoulder. Seen with Dr. Conrad Bell, of Waltham, Mass. Examination shows no power in any muscles of thigh or lower leg. Leg lies outwardly rotated, knee and ankle flail. Hip partially subluxated in the lying position, and by adduction and flexion can be easily slipped out of the joint upward and backward on to the ilium. X-rays of this case are not characteristic owing to the age of the patient and the short duration of the paralysis, but I venture to state that in time they will show the usual deformities and bone changes. She was fitted with a caliper splint, and can now walk well with a moderate limp.

CAse 13. C. Mac B. Boy. Age seven and one half. Paralysis of whole left leg since Oct. 1, 1908. On April 12, 1911, the condition was as follows: One inch shortening of whole leg, no power in lower leg muscles except very slight in flexors of toes, ankle practically flail, considerable atrophy of calf and thigh, walks with a limp, but hip does not dislocate in weight bearing. Trochanter is two inches above Nélaton's line on the left. Fair power in all thigh and hip muscles, but considerably less than on right, which is normal. No contractures. The hip can be dislocated upward and backward easily by flexing and adducting the thigh. $\mathrm{X}$-rays taken with the hip in and out of the acetabulum show the usual changes in the femur and acetabulum. The x-ray taken of the hip in place shows a marked increase in the distance between the articular surfaces of the joint, and the one taken with the hip out shows a very considerable separation, with the head more behind than above the acetabulum. There is evidently considerable relaxation of the joint capsule.

The boy complains of discomfort in the region of the hip-joint, and states that the leg gets tired easily and feels unstable. He occasionally feels the hip slip in and out while walking. He gets about easily with only a short brace to support his ankle. CAse 14. W. M. Boy. Age eight. Has had since one year of age, left leg. Came to hospital first paralysis of both legs for five years. Examination in March, 1911, showed that the left hip was subluxated, all muscles of the left thigh and hip-joint were weak but functionated; leg lay in outward rotation. With the child lying on his back with the leg in the fully extended position, but outwardly rotated, the trochanter could be felt well behind the head, which pointed upward and forward, and could easily be felt just outside the line of the anterior superior spine. In the right leg paralysis was limited to the lower leg. In walking the left leg was so short that in order to get it to the ground abduction of the leg on the pelvis took place, so that the head of the femur was held in the acetabulum and did not dislocate. When lying on the back, the head could be slipped in and out of the acetabulum easily. X-ray on March 17, 1911, with the left leg flat on the table, showed a coxa valga, a shallow acetabulum with the head of the femur about half out of the joint. There was considerable space between the articular surface of the femur and the acetabulum, suggesting a relaxation of the capsule and ligaments. (See Figure No. III.) There is marked atrophy of the shaft and trochanters. The head of the femur is about half outside the upper edge of the acetabulum. With the thigh flexed and adducted, the head is well out, up and back. The lower portion of the acetabulum was especially shallow, with the characteristic atrophy or absorption of the upper edge or shelf.

CAsE 15. M. M. Girl. Paralysis for past ten years. No voluntary motion of either hip or knee, slight flexion and extension of toes of right foot. Paralysis of left upper arm and paralysis of legs is practically complete. Examination on March 17, 1911, showed the following conditions: A very large, well-developed and rather obese child. Can walk very slightly by means of double calipers, a leather jacket and crutches. Both legs flaccid, apparently both hips are dislocated upward and backward. The right hip can be reduced and redislocated easily, with considerable excursion of the head of the femur. The left hip is apparently subluxated clinically, but is not as relaxed as that on the right. Marked contraction of tensor vaginæ femoris muscles.

The x-rays, taken with the child on her back, show a very marked tilt forward of the pelvis, so that both acetabulæ are directed downward and backward. Both acetabulæ are very shallow and flattened. The right hip is apparently in the acetabulum. The left hip is dislocated upward and backward, with a moderate coxa valga. Both legs are adducted and flexed.

CASE 16. A. D. Boy. Paralysis of right leg for past seven years. The capsule of the hip-joint is very much relaxed, and the hip can be dislocated on to dorsum of ilium with very slight effort. The leg can be voluntarily lifted off of the table with the child lying on his back. He can flex and extend the lower leg on the thigh. Good power in abduction, outward rotators strong, inward rotators paralyzed, adductors strong, no contraction of tensor vaginæ femoris. Gluteii paralyzed on right. Cannot use leg to any extent, and has to be supported in walking. X-ray taken January, 1911, shows the usual dislocation upward and backward, with a moderate coxa valga, atrophy and shallow flattened acetabulum. Pictures taken with the leg rotated first inward and then outward show that there still persists a moderate angle to the neck of the femur.

This boy was sent into the hospital with the idea of doing an arthrodesis to the hip-joint, but it was thought advisable to operate on his foot and lower leg before attempting a fixation of his hip-joint.

CASE 17. M. C. Girl. Age fifteen. Paralysis 
when three and one half years of age. The dislocation of the hip was first noticed in 1908. In 1911 the condition was as follows: Marked contraction of the tensor vaginæ femoris and adductor group. The lower leg can be flexed on thigh, but cannot be extended. The thigh cannot be extended nor the lower leg lifted. No power in abduction or flexion of thigh, no power in inward or outward rotation. The leg is permanently flexed about thirty degrees, and adducted about fifteen degrees. The head of the femur, which was first thought to be the great trochanter, is two inches above Nélaton's line. She walked with a caliper splint to support the knee and leg in weight bearing. There was also a bad limp and drop to the left side in weight bearing. She was advised to have an arthrodesis for fixation of the hip-joint. The x-rays of the hips showed a dislocation with a marked mushroom effect of the head and neck, with practically no acetabulum. Considerable atrophy of the shaft of the femur and left side of the pelvis. It was not possible to reduce the dislocation on account of the filling up of the acetabulum. The head is well outside of the acctabulum, and in weight bearing makes a considerable upward excursion, due to relaxation of the capsule and ligaments. (See Figure No. 4.)

Case 18. L. K. Boy. Age thirtcen. Paralysis of left leg since June, 1900 , age two. At that time was unable to raise leg or move toes. Could flex thigh slightly and could rotate leg in and out. Knee and ankle-joints practically flail. In April, 1908, was operated upon, and the ilio-tibial band was isolated and lengthened by silk, which was inserted below the patella to take the place of the quadriceps tendon. There was about two inches shortening of the leg. The boy walked with a bad limp and had to wear a caliper splint. In walking, the head dislocated at each step. The head of the femur could be easily dislocated and replaced, the head sliding upward and backward. On Dec. 20, 1910, an arthrodesis was done on the left hip-joint for the purpose of fixing it in the acetabulum. The joint was opened by the usual straight incision extending downward and outward from the anterior superior spine for a distance of about five inches.

The capsule was noticeably stretched. This was divided by a straight incision and the joint opened. The dislocation of the head of the femur was difficult, owing to the neck of the femur being in a line with the long axis of the shaft. The head was large and somewhat mushroom in shape. The upper and inner articular surface was chiseled off, including portion of the attachment of the ligamentum teres. The upper and inner part of the articular surface of the acetabulum, which was very shallow, was curetted. The head was then replaced in the acetabulum, care being taken to bring the denuded surfaces in contact. The relaxed capsule was quilted with kangaroo tendon so as to make it as snug about the head as possible, and the wound was closed. A plaster was applied from the waist to the toes with the leg abducted about fifteen degrees. A pathological report of the portion of the articular surface removed from the head of the femur showed nothing abnormal. The articular portion which was removed included a small portion of the epiphyseal line.

This boy was seen about twelve weeks after the operation. There was very slight mobility present in the hip-joint and, therefore, the plaster was reapplied.

The x-rays taken before operation show the head in the acetabulum and also dislocated upward and backward. There was a very marked coxa valga, with marked atrophy, especially of the great trochanter. There was the usual deformity of the acetabulum. After operation the head was seen to be in the reformed acetabulum, with a good shelf of bone above the head. At the time the arthrodesis was done on the hip further tendon transplantation and lengthening were done about the knee-joint. (See Fig. No. 5 , before operation.)

This boy was seen on May 8, 1911. The condition was as follows: The left leg was about two inches shorter than the right. It was fixed in about fifteen degrees abduction, with about five degrees outward rotation. The hip was in place and solid. He was fitted with a caliper splint, and is to be allowed to use leg and have massage.

Case 19. H. Z. Girl. Age thirteen. Paralysis, left leg, since two years of age. Arthrodesis of left ankle in September, 1904, on account of a flail joint. It was first noticed that the hip was dislocated in July, 1907. In November, 1908, a plaster spica was applied with the leg in marked abduction, for the purpose of fixing the head of the femur in the acetabulum. This was worn for four months without any benefit. X-rays taken before and after the application of the plaster show that the dislocation was not completely reduced, on account of the shallow acetabulum.

Examination Nov. 21, 1910, showed a large overgrown girl. Locally what was supposed to be the trochanter was found to be the head of the femur one and five-eighths inches above Nélaton's line. She walked with a marked limp and drop to the left. There was two and one-half inches shortening of the left leg. The paralysis of the leg was complete, with the exception of considerable contraction of the tensor vaginæ femoris muscle and the adductors. The leg was generally flexed and adducted. X-ray showed the hip well out, above and back of the acetabulum. What I supposed to be the trochanter turned out to be the articular surface of the head of the femur. There was a very flat shallow acetabulum, a marked coxa valga and marked atrophy of the pelvis and shaft of the femur. The x-rays also demonstrated the improbability of a successful and permanent reduction without an open operation for the deepening of the acetabulum to receive the head of the femur.

An operation was advised and accepted, and on the 6th of December an arthrodesis of the hip-joint was done. Incision four and one-half inches long over hipjoint, carried down to joint capsule, which was very much relaxed and stretched. With the leg adducted flexed and rotated outward, the head was dislocated. The acetabulum was found to be very shallow and elongated. There was a thick band of relaxed capsule over which the head of the bone slipped during the process of subluxation. There was no apparent abnormal twist to the neck of the femur. The top and inner portion of the articular surface of the head of the femur was chiseled off. The upper and inner portion of the acetabulum was thoroughly curetted and reshaped with a chisel. The head was then replaced, with the denuded areas in opposition, and the capsule was pleated and stitched with kangaroo tendon. The wound was then closed in the usual way. The tensor vaginæ femoris was divided through a separate incision, and the leg put up in plaster in about twenty degrees abduction.

This case was seen on March 22, 1911. There was very slight motion present in the hip-joint, no tenderness or pain on attempted manipulation. The plaster was omitted and a flannel bandage applied to be worn for several weeks. (See Figure No. 6.)

On May 8, 1911, this case was seen again. She has been walking without crutches or splint for about two weeks. Can bend knee to a right angle. Hip solid. No difficulty in sitting down. No pain or discomfort. About two inches shortening. 
Seen again in June, 1911. Much improvement. Walks well, but slowly. States that she feels better than before operation, and that the condition of the leg is much more satisfactory. States that she is able to walk better and with less discomfort.

Case 20. F. N. Boy. Age twelve. Infantile paralysis ten years ago, affecting both legs. In the left leg the paralysis was confined almost wholly to the lower leg muscles. On the right the leg was flail at the hip, knee and ankle. There was no power in the foot muscles or lower leg muscles. Fair power in gluteii on both sides. Could not extend, flex or abduct thigh on body. The adductors were paralyzed, and the tensor vagina femoris on the right was markedly contracted. The right hip could be easily subluxated upward and backward. The trochanter was one inch above Nélaton's line, with about one inch shortening of the leg as a whole. He could walk only by means of caliper splint on right, and complained of a limp on the right, with a rather useless leg.

$\mathrm{X}$-rays were taken, with the boy on his back, of the hip in and out of the acetabulum. The subluxation was marked, and the capsule was apparently much relaxed. The slipping of the head over the acetabular rim could be easily felt. The x-rays showed the usual coxa valga, with a shallow flat acetabulum, so that even with the head in place it was partially outside of the acetabulum. There was the usual bony atrophy of the shaft. The upper shelf of the acetabulum was markedly absorbed, so that in weight bearing the head slipped out of the socket, even with the leg abducted. An operation to fix the hip in the socket was advised and accepted. This was done on Dec. 9, 1910.

Operation. - An incision about five inches long was made, extending downward and outward from below the anterior superior spine and carried between the tensor vaginæ femoris and the gluteus medius to the joint capsule. The capsule was found much relaxed. This was opened, exposing the joint. The head was easily disarticulated by flexing and adducting the thigh. A portion of the upper and interior surface of the articular head was removed and reshaped with a chisel to fit snugly into the acetabulum, which was not curetted. The joint was then closed. The acetabulum was not as deep as normal. There was only a very slight shelf at the upper part of the acetabulum, which was in appearance the remainder of the normal upper lip. There was not a complete coxa valga, and there still persisted a slight angle to the neck of the femur. The great trochanter was seen to be very much atrophied and slightly elongated at its upper end. The tensor vaginæ femoris muscle was divided through a separate incision, and the upper end of the lower portion was fastened by means of kangaroo tendon into the fascia on the outer side of the ilium over the site of the trochanter. Before closing the larger wound the capsule was quilted with kangaroo tendon to take up the slack. The leg was then put up in plaster in an abducted position, from toes to thorax.

This case was in plaster until March 8,1911 , when there was found to be the slightest possible motion present at the hip. He was given crutches and told to walk on the leg. An x-ray taken on this date showed the head of the femur well engaged in the acetabulum.

This case was seen again on May 8, 1911. He could walk with crutches and the caliper splint on the right lcg. The hip was firmly fixed. As he had no power at the knee or ankle, the stiffness of the hip gave him some trouble in learning to swing the leg, which I expect he will be able to overcome. He will eventually have to

\begin{tabular}{c|l} 
have an arthrodesis done at his ankle-joint. & slight adduction of the \\
CASE 21. E. N. G. Boy. Age eleven and one-half & shortening of the legs.
\end{tabular} years. Paralysis since age of two years. Has never had any treatment. Unable to walk. Seen March, 1911. Left leg flexed acutely at thigh and knee, abducted and rotated out so that it lies along the side of the child's body. The head of the femur can be felt well downward, forward and inward, so that it apparently lies well towards the anterior portion of the obturator foramen. The adductors and all of the other thigh and hip muscles are paralyzed, except for the contracted tensor vaginæ femoris, which is unopposed. The foot is in a position of paralytic talipes equinovarus. It was not possible to correct this deformity or to reduce the dislocation without an open operation, which was advised.

CASe 22. E. B. Boy. Age nine. Paralysis at about two years and a half. Has never had any treatment. When first seen was unable to walk or to help himself. Double paralytic talipes equinovarus. Flexion contractions at both knees. Both arms paralyzed, with deformity contractures. Scoliosis. Could not turn himself in bed or feed himself without great difficulty. Seen March, 1911. Both hips dislocated. Right more relaxed than left. Marked contraction of adductors and tensor vaginæ femoris on both sides. Double iliac dislocation. The child is now learning to walk with a walking frame, the contractures having been partially overcome by means of successive plaster bandages.

Case 23. S. B. Boy. Age six. Has had infantile paralysis of right leg since one year old. Examination March, 1911, shows the right leg abducted and flexed at the hip, the right knee is flexed by contraction of the thigh flexors, no power at hip in extensors or adductors. Rectus femoris paralyzed. The head of the femur lies above and behind the acetabulum on the ilium.

CASE 24. E. J. J. Boy. Age nine. Infantile paralysis since one year of age, left leg. Seen March, 1911. Left hip dislocated up and back, marked contraction of adductors and tensor vaginæ femoris and flexors of knee.

Case 25. J. C. Boy. Age sixteen. Paralysis for fourteen years, right leg. Examination shows complete loss of power in external rotators, very slight power in rectus femoris, cannot extend lower leg fully as thigh. Flexors of leg on thigh weak. Fair power in inward rotators, slight permanent flexions of knee. Leg three inches short. Trochanter well above Nélaton's line. Leg lies inwardly rotated, adducted and flexed. Head of femur dislocated and lies above and behind trochanter well above acetabulum. Can be replaced in acetabulum, but does not stay in place. Marked contraction of tensor vaginæ femoris and adductors. Lower leg shows considerable paralysis, with toe drop. Walks with crutches, but can walk without them by supporting thigh with hand, to prevent flexion of knee.

Case. 26. Boy. Age ninetecn. Paralysis for past fifteen and one-half years. Until he was about nine years of age, he went about on his hands and feet, " all fours." The paralysis was very extensive, involving the extensors of both lower arms, the back and abdominal muscles and the legs. The paralysis of the legs was practically complete, there being only very slight power of flexion in the toes on the left foot and slight power in the flexors of the lower leg on the thigh of the right leg. All of the gluteii muscles on both sides are paralyzed. He can walk and get about only by means of a jacket, caliper splints and crutches. The hip on the left is subluxated, the head of the femur being displaced upward and backward. There is very slight contraction of the tensor vaginæ femoris, with slight adduction of the leg. There is about three inches 
A study of these cases shows that out of the twenty-six reported there is only one which is of the so-called infrapubic type, while the other twenty-one are dislocations on to the ilium, or so-called post-iliac dislocations. The practically constant presence of the unopposed contractions of the adductors and tensor vaginæ femoris muscles show the reason of the prevalence of this posterior type. If, however, these two muscles are allowed to continue to contract unopposed, the dislocation may become infrapubic or obturator in type, as shown by Case 21. As the leg continues to flex, adduct and outwardly rotate, the head of the femur naturally follows a definite path from above the acetabulum to below and internal to it.

The frequent escape of these two muscles from being involved in the paralysis is probably to be explained by the fact that the adductors are supplied by an upper branch of the lumbar plexus, namely, the obturator nerve, although this does not hold true of the nerve supply to the tensor vaginæ femoris, which is the superior gluteal nerve. It is probable that the medullary centers of these two nerves are at higher or different levels in the cord, probably in the upper part of the lumbar enlargement, than the centers of the nerves which supply the other muscles of the thigh and hip, and so escape destruction.

This theory seems probable in view of the fact that the gluteus medius and minimus are almost always paralyzed, while the tensor vaginæ femoris escapes, yet they are all supplied by the superior gluteal nerve. The examination of the nerves at the origin of the plexus will give us the explanation of this paradox. In the multiple origin of the nerve in the plexus we find the possibility of fibers arising from different centers for the different muscles which are innervated by the same nerve.

It is evident then that these unopposed contractures play a very important part in producing a subluxated hip-joint, in combination of course with the usual atrophy of bone and soft parts due to the paralysis. When all of the hip and leg muscles are paralyzed, the dislocation is not so apt to occur, not so quickly produced, nor so extensive.

\section{TREATMENT.}

A flail leg following complete paralysis is not a very useful member, and an arthrodesis of one or more joints often makes it of use as a stilt or crutch, by means of which the patient can get about more easily than before the operation. Splints are cumbersome, easily broken and heavy, and any means by which they can be discarded to the patient's ultimate benefit is of course justified.

The stiffening of the ankle, knee and hip in a single case is a good deal to do, and is not advisable. The ankle and knee may often be stiffened at separate times with considerable benefit. The hip, if badly subluxated and causing symptoms of pain and discomfort, may have an arthrodesis performed, and the ankle on the same leg may also be stiffened. To do an arthrodesis of the hip-joint and knee-joint on the same leg makes the leg difficult to move or swing and is not a justifiable procedure. Often an arthrodesis of the knee alone, if the hip is not badly subluxated, is enough to steady the leg for weight bearing. However, each case is an individual problem and should be studied and worked out according to the conditions found and the prospects for the relief possible to obtain by any given operation or operations. Often the knee and ankle can be strengthened by means of silk ligaments and tendon transplantation, following an arthrodesis on the hip-joint.

Paralytic dislocations of the hip will not stay in place after simple replacement, even following fixation in plaster, for plaster casts cause atrophy and, therefore, simply increase an already existing condition. I question whether simple quilting of the capsule in its atrophic state would be sufficient to hold the head of the femur in its shallow atrophied acetabulum, when weight bearing and use was again applied to it, and I believe that in every case where the femoral head is definitely dislocated, an arthrodesis, complete or partial, must be done in order to secure a stable and permanent joint, without danger of recurrence.

The contraction deformities must always be overcome either by means of gradual stretching, in plaster or by extension in bed, or, if too severe, by means of an open incision and complete division. In cases where there is much muscle tissue involved, it is better to resect a portion of the muscle itself to prevent subsequent contractions.

In attempting to stretch the contractions of the adductors and tensor vaginæ femoris muscles, the leg must be extended and abducted as much as possible at all times, and the subluxation of the femoral head must be kept reduced if possible so that a bony point of resistance is opposed to the forces used to stretch the muscles. Otherwise the resistance will be on the upper part of the joint capsule, which will consequently stretch more and more, and so allow the femur to move upward as a whole, without any great gain being made in stretching the contractures. Some slight gain may be made, however, even if the head is not engaged in the acetabulum, on account of the muscle atrophy induced by the application of the plaster of Paris cast, or by means of slight stretching taking place in the muscles from a longcontinued pull, causing the muscle to tire. These slight gains are rarely permanent and tend to recur. I believe that it is better in the severe cases to divide the contractures through an open incision if any hope of a permanent result is to be entertained.

In the early stages, before the head of the femur is definitely subluxated, it is not wise to neglect any contractures. Careful and thorough stretching, and even division, at this time will often prevent a subsequent subluxation of the hip, which when definitely established is a difficult condition to combat, and if at all severe usually leads to marked disability, following which much more severe operative procedures have to be under- 
taken than would have been necessary provided the contractures had been properly stretched at first.

Permanent changes in the head and neck of the femur and acetabulum due to atrophy are practically constant, and are as follows:

Coxa valga. This is an increase in the angle of the neck of the femur, often to such an extent that the long axis of the neck seems to be in the same plane as the long axis of the shaft. This condition is usually present, and varies only in degree. It is apparently due to the effects of gravity, that is, the weight of the dependent leg tends to straighten out the bone and is merely the result of one force overcoming another and lesser one.

This condition is, of course, assisted materially by the existent paralysis of the muscles about the hip, which offer no resistance to the "dangle leg." The marked atrophy of the bony points of attachment of the paralyzed muscles, such as the greater and lesser trochanter, is also noteworthy.

The shallow, elongated and flattened acetabulum is practically always constant. In some cases it is seen to be higher than the normal one on the opposite side. This is often due to the absorption or wearing away of the upper lip or shelf of the acetabulum and the accompanying atrophy of the whole side of the pelvis. In some cases it has become so shallow and flattened that any attempt at reduction of the hip is an impossibility without first deepening the acetabulum by means of an operation.

\section{PROGNOSIS.}

The prognosis in these cases is the important and interesting thing and was the question which I wanted to answer for myself, and for which I began this research. In looking over this collection of cases they naturally divide themselves into three classes:

Class I. Cases 1 to 5 inclusive.

Those cases where the atrophic changes in the joint have become evident, but where they have not gone on to such an extent as to have produced a subluxation. In these cases there is usually also moderate contraction of the tensor vaginæ femoris muscle, and possibly the adductors. It is obvious that the only thing to do under these conditions is to stretch the contractions. This may be done by means of frequently applied plaster of Paris spicas, or by means of traction applied with the leg in an abducted and extended position in bed. After these contractions have been corrected, a splint, such as a caliper, may be worn, with a pelvic belt, as an aid in walking and preventing further contractions. To overcome any limp on account of a short leg, a lift may be worn on the shoe on that side. It is necessary to watch the case carefully and to see it at regular intervals, for these contractions are very apt to recur. As a rule, however, the cases in general, if seen during their first stage, do well if kept under treatment, and do not tend to get worse. Massage and muscle training are essential.

Class II. Cases 6 to 19 inclusive.

These cases represent the less severe types of subluxated hip. That is, the disability is not great and the dislocation is not severe and easily reducible. The hip does not always slip out in walking or weight bearing, and the contractions are not very marked. They represent the later stages of the cases in Class I, the early treatment having been neglected. Without treatment they will probably get worse, that is, develop more severe contraction deformities, and consequently a greater degree of dislocation at the hip, with more subsequent disability, which will gradually tend to increase. The treatment of these cases should be directed towards reducing the muscular contractions and replacing the dislocated hip.

Class III. Cases 14 to 26 inclusive.

The cases in this group include the more severe types of paralytic dislocations which occur, and usually are the result of lack of care and treatment. They may and usually do follow the conditions seen in Classes I and II, and have probably been without adequate treatment.

An operation is usually necessary, especially to overcome the muscle contraction, and an arthrodesis of the hip with a deeping of the acetabulum is often advisable, provided a stable joint is to be expected. The complications are usually so severe that the problems in each case are perplexing, and it is difficult to lay down any hard and fast rules for the conduct of the cases as a class. Each case has to be treated according to.its needs and expectations. The tendency without treatment is towards greater deformity, that is, a shorter leg, more flexion, outward rotation and abduction and a greater degree of subluxation. The longer a case is untreated, the more difficult it is to apply the remedy. When the leg becomes much shortened it may be impossible to reduce the dislocation and get the leg down where it belongs, on account of the permanent contraction of the nerves and vessels. Then if a stable hip is desired, a resection of the upper end of the femur is all that can be done, followed by an arthrodesis and a resulting permanent short leg.

\section{CONCLUSIONS.}

I. Subluxations of the hip joint are not uncommon conditions following paralysis of the thigh and peritrochanteric muscles.

II. The most important factors in their production are the unopposed contractions of the non-paralyzed muscles.

III. The two muscles which escape the paralysis most frequently are the adductors and the tensor vaginæ femoris.

IV. The most common type of subluxation is upward and backward on to the ilium.

$V$. The changes from atrophy in the femur and acetabulum are practically constant, and consist of a coxa valga and a shallow, flattened and elongated acetabulum.

VI. The treatment should be directed towards correcting the contractions and reducing the dislocation of the hip.

VII. The muscles may be elongated by stretch- 
ing, by means of plaster casts, bed traction or division.

VIII. The best method of keeping the hip reduced is by means of an arthrodesis, although this is not practical in all cases.

IX. Without treatment the tendency is towards progressive deformity.

$\mathrm{X}$. With treatment the conditions may be permanently helped.

XI. Complicating conditions make a total cure impossible, but a more useful leg should be assured under proper treatment.

XII. Walking after arthrodesis is often improved.

XIII. In some cases splints may be altogether discarded.

XIV. It is not advisable to do an arthrodesis on both hips, or on any joint other than the hip and ankle on the same leg.

\section{REFERENCES.}

1 Reclus: Rev. de Méd. et Chir., 1878, p. 176

2 Karewski: Ann. Surg., vol. x, 1889, p. 226.

3 Stimson: Fractures and Dislocations, vol. ii, 1899

$4 \mathrm{v}$. Nicoletti: Zentralbl. für Chir. Mech. Orthop., January, 1910, p. 44 .

s Vulpius: Münch. Med. Wochenschr., 1908, s. 7

6 Böcker: Verhandl. Deutseh. Gesellsch. für Orthop. Chir., bd. xxiv, April, 1909.

${ }^{7}$ K. Cramer: Arch. für Orthop. Mech. u. Unfallchir., bd, ix, Heft 1, 1910.

8 Reidinger: Ibid., bd. viii, Heft 3, 1910

9 Lovett and Iucas: Jour. Am. Med. Asso., vol. li, Nov. 14, 1908. BIBLIOGRAPHY.

Verneuil: Bull. de la Soc. de Chir., Gaz. de Hôp., no. 70, 1866.

Tubby: Orthopedic Surgery, 1896.

Bradford and Lovett: Ibid., 2. ed., 1899.

Keen's Surgery, vol. ii, 1907.

Taylor, H. L.: Orthopedic Surgery, 1909.

Sayre: Ibid., 1883.

Hoffa: Ibid., 1905 .

Holtzmann: Virchow's Arch. für Path. Anat. u. Phys. u. Phys. F. K.

Med., bd. 140, 1895 .

Rich, E. A.: Northwest Med. Jour., Seattle, vi, p. 335, 1908.

Martin: Zeitschr. für Orthop. Chir., bd. x, Heft 4, 1902.

International Textbook of Surgery, 1900 .

Gray's Anatomy, Pick's edition, 1897.

Lombard and Abbott: Am. Jour. Physiol.,vol.xx, no. 1, October, 1907 . Extrait du Congrès Français de Chirurgie, 23d session, Paris, 1910.

\section{INTERSTITIAL GINGIVITIS.*}

BY EDWARD C. BRIGGS D.M.D, M.D., BOsTON.

THE invitation to a practicing dentist to lecture before graduates of medicine implies that what has always been a fact is now recognized, i. e., the intimate relation of dentistry to medicine, and the importance of the realization of that relation by the medical practitioner.

It is my intention in this lecture to call your attention to interstitial gingivitis, - the importance of its recognition by the physician, - and to show his field of usefulness in its treatment.

To begin with, let me explain why I adhere to this term. I do not object to calling the disease " Riggs' disease." Whatever honor redounds to a man in having his name given to a disease surely belongs to Dr. Riggs in this connection. $\mathrm{He}$ is on record as having first described and first treated the trouble, in 1846. And it is really remarkable the way he covered the subject and how little he has left for us to improve upon. The strange thing is the long time it has taken the profession as a whole to follow in the footsteps of his teaching. Even now, after forty years, there

* Address delivered before the Harvard Summer School of Medicine July 21, 1911 . are many who make no attempt to prevent or cure the disease and of those who do so attempt many are faint hearted and give up the fight without half trying.

Acting on the theory that "Riggs' disease" was not a sufficiently scientific term, an attempt was made to attain the higher plane and "pyorrhea alveolaris "was put forth. For this term I have no use whatever, and condemn it in no uncertain words. It is as if typhoid fever were called hemorrhage of the bowels and no one recognized or treated a patient as sick until the hemorrhage appeared. As it would be pretty late to save a typhoid patient after the disease had destroyed the integrity of the intestine, so is it difficult to cure "interstitial gingivitis" when the average practitioner has found and diagnosed what he calls " pyorrhea alveolaris." Pus flow is a symptom that comes late in the disease. It comes after the deposition of the nodules, and that is not the first thing that happens.

The duty, then, of the practitioner is to diagnose the disease in the earliest stage possible, although he is to treat it in all stages with a reasonable hope of cure in almost every case. Every patient, at any age, when being examined for caries of the teeth, should be just as carefully examined for " Riggs' disease." Examine around all the teeth for pockets between gum and tooth, testing with considerable force, as many pockets where pus has not begun to flow are extremely blind, there being little evidence to the eye that there is any trouble there. Note the cases of simple recession of the gum, especially those where the gum margin is " corded," as if a drawing string had been run through. These are cases of " interstitial gingivitis," cases where treatment will be most effective and curative. If the gum is dark mahogany color at the tooth margin, or pus is exuding, the diagnosis is made by the eye alone.

What do we find in these pockets? If the disease is far advanced, we find great roughness of the root due to deposits of lime salts from the serum of the blood which has escaped from the ruptured blood vessels of the degenerated gingival tissues. G. V. Black, the foremost worker in research in our specialty, has lately given his attention to this disease, and I shall quote freely from an article read by him in January of this year. "The gingivæ include all of the tissue which is superimposed upon the margin of the alveolar processes and attached to the teeth. They rise about the teeth, more or less investing the crowns and terminating in a free border. It is the preservation of this tissue, and more particularly of the integrity of this free border, that calls for constant watchfulness and skillful work on the part of the dentist." It is the recognition of this fact that has more than all else brought about the Black system of contouring teeth and the redoubled (and what seems to the uninformed unreasonable) efforts in the regulation of teeth, orthodontia as it is called.

The attachment of the soft tissues to the alveolar process is similar to other attachments of soft tis- 